\title{
Criação e utilização de tecnologias para enfrentamento da COVID-19 frente ao período de pandemia
}

\author{
Creation and use of technologies to combat COVID-19 during the pandemic period \\ Creación y uso de tecnologías para enfrentar el COVID-19 durante el período pandêmico
}

Recebido: 20/01/2021 | Revisado: 23/01/2021 | Aceito: 27/01/2021 | Publicado: 04/02/2021

Antônio Diego Costa Bezerra

ORCID: https://orcid.org/0000-0002-2441-2961

Centro Universitário Unifanor, Brasil Fundação Oswaldo Cruz, Brasil

E-mail: diegocostamjc@gmail.com

Kamila de Castro Morais

ORCID: https://orcid.org/0000-0002-3564-7993

Universidade Regional do Cariri, Brasil

E-mail: kamilacastromorais@gmail.com

Mariany de Alencar

ORCID: https://orcid.org/0000-0003-2959-6445

Universidade Federal do Piauí, Brasil

E-mail: mariany.alencaar@gmail.com

Romulo de Oliveira Sales Junior

ORCID: https://orcid.org/ 0000-0002-4638-7971

Centro Universitário UNINOVAFAPI, Brasil

E-mail: romulojr_99@hotmail.com

Ana Luiza Linhares Beserra Machado

ORCID: https://orcid.org/0000-0002-7585-9739

Faculdade Princesa do Oeste, Brasil

E-mail: analuizabeserra@hotmail.com

Rita de Cássia Loiola Alves

ORCID: https://orcid.org/0000-0002-0241-3939

Universidade Federal do Piauí, Brasil

E-mail: kassia.loiola@gmail.com

Debora Ellen Sousa Costa

ORCID: https://orcid.org/0000-0003-4205-8801

Universidade Federal do Maranhão, Brasil

E-mail: deborasousacosta@outlook.com

Maria Andressa Gomes de Lima

ORCID: https://orcid.org/0000-0002-0543-4399

Centro Universitário INTA - UNINTA, Brasil

E-mail: andressagomesdelima47@gmail.com

Lívia Sayuri Félix Mendes

ORCID: https://orcid.org/0000-0001-7166-7708

Centro Universitário INTA-UNINTA, Brasil

E-mail: livya.mendessp@gmail.com

Daniel Coutinho dos Santos

ORCID: https://orcid.org/0000-0002-6230-9842

Universidade Federal do Maranhão, Brasil

E-mail: daniel.coutinho@discente.ufma.br

Luciano Santos da Silva Filho

ORCID: https://orcid.org/0000-0003-2325-3716

Escola de Saúde Pública do Ceará, Brasil

E-mail: lucianofilhofisio@gmail.com

\begin{abstract}
Resumo
$\mathrm{O}$ artigo teve como objetivo evidenciar na literatura científica informações acerca das tecnologias e ferramentas utilizadas no enfrentamento da COVID-19. Trata-se de uma revisão de literatura, onde o levantamento bibliográfico foi realizado através dos bancos de dados: Scientific Eletronic Library Online (SCIELO), Sistema Online de Busca e Análise de Literatura Médica (MEDLINE), ScienceDirect e Business Source Complete (EBSCO). Os critérios de inclusão dos trabalhos foram: artigos publicados entre 2019 e 2020, textos completos, disponíveis na íntegra, no idioma português, inglês e espanhol. Para exclusão: monografias, teses, artigos de revisão, literatura cinzenta e artigos repetidos, resultando ao final 13 artigos para compor a pesquisa. Os estudos trouxeram abordagens amplas e diferentes nas pesquisas sobre tecnologias, inovações e COVID-19, como por exemplo: aplicativos móveis,
\end{abstract}


telessaúde, desenvolvimento de máscaras de proteção, treinamento seguro e eficaz para profissionais da saúde, etc. A pandemia e a crise no setor saúde evidenciada com a COVID-19 serviram de campo fértil para a criação e adaptação de tecnologias para auxiliar no enfrentamento dos casos, tornando-se fundamentais para a prática assistencial. Nesta perspectiva, surgiram ferramentas inovadoras para o combate à epidemia da COVID-19 em todo o mundo.

Palavras-chave: Controle de doenças transmissíveis; Infecções por coronavírus; COVID-19; Tecnologia em saúde.

\begin{abstract}
The article aimed to show in the scientific literature information about the technologies and tools used in combat with COVID-19. It is a literature review, where the bibliographic survey was carried out through the databases: Scientific Eletronic Library Online (SCIELO), Medical Literature Analysis and Retrieval System Online (MEDLINE), ScienceDirect e Business Source Complete (EBSCO). The inclusion criteria for the papers were: articles published between 2019 and 2020, full texts, available in full, in Portuguese, English and Spanish. For exclusion: monographs, theses, review articles, gray literature and repeated articles, resulting in 13 articles at the end of the research. The studies brought broad and different approaches to research on technologies, innovations and COVID-19, such as: mobile applications, telehealth, development of protective masks, safe and effective training for healthcare professionals, among others. The pandemic and the crisis in the health sector evidenced with COVID-19 served as a fertile field for the creation and adaptation of technologies to assist in coping with cases, becoming fundamental to the care practice. In this perspective, innovative tools have emerged to combat the COVID-19 epidemic worldwide.
\end{abstract}

Keywords: Communicable disease control; Coronavirus infections; COVID-19; Biomedical technology.

\title{
Resumen
}

El artículo tenía como objetivo mostrar en la literatura científica información sobre las tecnologías y herramientas utilizadas para hacer frente a COVID-19. Se trata de una revisión de la literatura, donde se realizó el relevamiento bibliográfico a través de las siguientes bases de datos: Scientific Eletronic Library Online (SCIELO), Medical Literature Search and Analysis Online System (MEDLINE), ScienceDirect y Business Source Complete (EBSCO). Los criterios de inclusión para los trabajos fueron: artículos publicados entre 2019 y 2020, textos completos, disponibles íntegramente, en portugués, inglés y español. Por exclusión: monografías, tesis, artículos de revisión, literatura gris y artículos repetidos, resultando 13 artículos al final de la investigación. Los estudios aportaron amplios y diferentes enfoques de investigación sobre tecnologías, innovaciones y COVID-19, tales como: aplicaciones móviles, telesalud, desarrollo de máscaras protectoras, formación segura y eficaz para profesionales de la salud, etc. La pandemia y la crisis del sector salud evidenciada con el COVID-19 sirvió como un campo fértil para la creación y adaptación de tecnologías que ayuden al afrontamiento de los casos, volviéndose fundamental para la práctica asistencial. En esta perspectiva, han surgido herramientas innovadoras para combatir la epidemia de COVID-19 en todo el mundo.

Palabras clave: Control de enfermedades transmisibles; Infecciones por coronavirus; COVID- 19; Tecnología biomédica.

\section{Introdução}

O novo coronavírus (SARS-CoV-2) é o responsável pela doença conhecida como COVID-19, na qual a mesma ganhou destaque após ser reconhecida sua alta capacidade de transmissão e letalidade, devido seu caráter de síndrome respiratória aguda grave. Inicialmente registrados casos na China e, posteriormente, chegando a diferentes regiões do planeta, atingindo mais de 500 mil pessoas em todo o mundo, caracterizando esse período como pandêmico (Júnior JHS et al. 2020).

Tal cenário, de certo modo, apresentou-se como inédito para a humanidade, sendo necessária uma resposta rápida e eficiente dos órgãos de saúde, com vista a resolutividade da incidência de novos casos, fazendo-se necessário repensar as condutas cotidianas e tomar providências pertinentes ao período vivido, reinventando assim o modo como ocorrem as relações interpessoais, as prioridades e a rotina da população (Coelho, et al., 2020).

Com isso, várias medidas de conscientização e contenção social têm sido propostas para conter o aumento exponencial desses casos, almejando sinalizar a população acerca da magnitude da pandemia de modo a respaldá-la quanto às condutas individuais e coletivas que devem ser realizadas para o controle desse vírus. Porém, para além dessa atividade, os órgãos de saúde devem se dispor a inovação, por meio da criação e utilização de tecnologias para o enfrentamento desses casos (Pereira, et al., 2020).

Assim, segundo Beaunoyer et al. (2020) a tecnologia e a inovação se tornaram ferramentas diferenciais e imprescindíveis em relação a pandemia, visto que as mesmas estão cada mais vez mais presentes na sociedade, salientando-se 
uma produção singular de informações e conteúdos técnico-científicos sobre a COVID-19, em decorrência da utilização dessas ferramentas.

Destarte, a utilização das tecnologias surge como uma forma de compartilhar informações sobre o tema, usadas por diferentes segmentos sociais - sociedade civil, governo, instituições privadas ou supranacionais -, como também auxilia a sociedade a repensar a produção de plataformas e ferramentas aptas para tornar possível a execução de procedimentos de monitoramento, controle e enfrentamento, de maneira prática e eficiente (Almeida, et al., 2020).

Quando relacionada a utilização dessas ferramentas no âmbito da COVID-19, enfatiza-se esta como sendo uma tendência mundial, focalizando no desenvolvimento e emprego de tecnologias e instrumentos digitais de informação que auxiliem no combate ao coronavírus, por meio de estratégias para acompanhamento e contenção da disseminação do vírus, possibilitando o rastreamentos de sintomas, contatos e deslocamentos, mostrando-se como efetivo para colaborar com estudos e previsões para o enfrentamento adequado desta e de futuras situações de saúde (Caetano, et al., 2020).

Diante da ânsia do exercício de uma assistência resolutiva e eficiente, observou-se a necessidade de pesquisar acerca das tecnologias utilizadas pelos profissionais de saúde no enfrentamento da COVID-19. Tal fato torna-se relevante, uma vez que as utilizações dessas ferramentas auxiliam diretamente no combate dos casos registrados, sendo indispensável sua utilização. Desse modo, o estudo tem como objetivo evidenciar na literatura científica informações acerca das tecnologias e ferramentas utilizadas no enfrentamento da COVID-19.

\section{Metodologia}

Constitui-se de uma revisão integrativa de literatura, de abordagem qualitativa e objetivo descritiva. A revisão integrativa, é a mais extensa abordagem metodológica relacionada às revisões, concedendo a inserção de estudos experimentais e não-experimentais para um entendimento completo do fenômeno pesquisado. É uma medida que possibilita a sinopse de entendimento e a inclusão da aplicabilidade de resultados de estudos importantes na prática (Souza, et al., 2010).

Em estudos qualitativos emprega-se a metodologia da pesquisa-ação, a qual busca evidenciar nas literaturas pertinentes meios para a resolução do problema exposto, a partir da prática reflexiva a qual se investiga através da leitura, busca e, posteriormente, aplicação dessas informações para obtenção e análise dos dados. Para isso, se torna necessário compreender a utilização das normas exigidas de acordo com o tipo de estudo (Pereira, et al., 2018).

Segundo Mendes et al. (2008) a revisão integrativa se baseia na pesquisa que utiliza recursos de dados de trabalhos já publicados, sendo caracterizado por 6 etapas específicas, ao qual a primeira etapa é a elaboração da questão norteadora, a segunda etapa é a busca ou amostragem na literatura, a terceira etapa é constituída pela definição das informações extraídas dos estudos selecionados, a quarta etapa é o processo de avaliação dos estudos incluídos, a quinta etapa é referente a discussão dos resultados e por fim a última etapa é a apresentação da revisão integrativa.

Dessa maneira, a construção da pergunta norteadora foi induzida a partir do tema Tecnologias para prevenção do COVID-19 e utilizou-se do anagrama PICOs resultando na pergunta: Quais as tecnologias utilizadas para o controle e enfrentamento da COVID-19 durante o atual período de pandemia? Contando assim, com os seguintes descritores: Controle de doenças transmissíveis; Tecnologia em Saúde; Infecções por coronavírus, indexados nos Descritores em Ciências da Saúde (DEC's).

A busca foi realizada nos meses de setembro a outubro de 2020 nas bases de dados: Scientific Eletronic Library Online (SCIELO) Sistema Online de Busca e Análise de Literatura Médica (MEDLINE), ScienceDirect e Business Source Complete (EBSCO). Foram realizados os seguintes cruzamentos: Controle de doenças transmissíveis and Tecnologia em Saúde and Infecções por coronavírus e Tecnologia em Saúde and Infecções por coronavírus. 
Os critérios de inclusão adotados foram: artigos publicados entre 2019 e 2020, textos completos, disponíveis na íntegra, no idioma português, inglês e espanhol. Para exclusão: monografias, teses, artigos de revisão, literatura cinzenta e artigos repetidos.

Para a identificação das publicações, realizou-se leitura criteriosa dos títulos, resumos e palavras-chaves de todas as publicações completas localizadas pela estratégia de busca e seleção a posterior e foram confrontadas com os critérios de inclusão e exclusão conforme estabelecidos. Desta forma as publicações foram utilizadas como objetos de análise, passando-se novamente por uma leitura criteriosa na íntegra guiada pela pergunta norteadora desta revisão.

Após leitura exaustiva de cada material selecionado, a fim de verificar a pertinência do estudo com a pergunta norteadora, verificou-se que 15 produções científicas serviram como fonte de dados para esta pesquisa. Para sistematizar a busca e seleção dos artigos (Figura 1) utilizou-se a recomendação do Preferred Reporting Items for Systematic Reviews and MetaAnalyses (PRISMA) (Moher, et al., 2009).

Figura 1. Fluxograma com representação de elegibilidade e inclusão de artigos na seleção dos estudos, 2020.

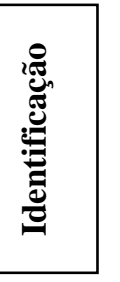

Estudos identificados por meio da busca bibliográfica nas bases de dados com o uso dos descritores: "controle doenças transmissíveis" AND "tecnologia em saúde" AND "infecção por corona vírus" com resultados: $\mathrm{Scielo}=3$, Medline $=22, \mathrm{Ebsco}=52$, ScienceDirect $=175$. Total $=252$.

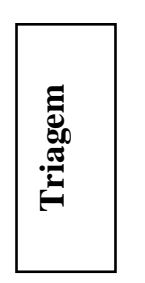

\section{$1^{\mathrm{a}}$ exclusão:}

Excluídos a partir dos critérios de inclusão $(\mathrm{n}=110)$

Duplicados $(\mathrm{n}=14)$

Estudos rastreados: Artigo completo,
disponíveis on-line, em Português, Inglês
e Espanhol e recorte temporal a partir de
$2019 . \mathrm{N}=142$.

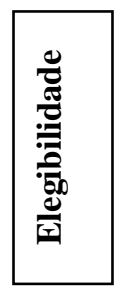

Estudos em texto avaliados para
elegibilidade: Leitura completa do artigo
$\mathrm{N}=13$.

\begin{tabular}{|l|l|}
$2^{\text {a exclusão: }}$ \\
Excluídos por título $(\mathrm{n}=47)$ \\
Excluídos por resumo $(\mathrm{n}=8)$
\end{tabular}

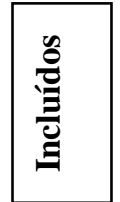

Amostra final: Estudos incluídos na síntese, os quais decorrem sobre as tecnologias utilizadas para o controle e enfretamento da COVID-19 durante o atual período de pandemia, após avaliação por pares às cegas. $\mathrm{N}=13$. 


\section{Resultados e Discussão}

Abaixo segue a Tabela $1 \mathrm{com}$ as principais informações dos artigos científicos que serão analisados.

Tabela 1. Apresentação dos artigos segundo título, autores, objetivo e a síntese dos resultados.

\begin{tabular}{|c|c|c|c|c|}
\hline $\begin{array}{c}\mathbf{N}^{0} \text { DO } \\
\text { ARTIGO }\end{array}$ & TÍTULO & AUTORES & OBJETIVO & SÍNTESE DOS RESULTADOS \\
\hline A1 & $\begin{array}{c}\text { Desafios e } \\
\text { oportunidades para } \\
\text { telessaúde em tempos } \\
\text { da pandemia pela } \\
\text { COVID-19: uma } \\
\text { reflexão sobre os } \\
\text { espaços e iniciativas no } \\
\text { contexto brasileiro }\end{array}$ & Caetano, et al. & $\begin{array}{c}\text { Discutir os espaços de } \\
\text { contribuição da telessaúde para } \\
\text { o enfrentamento da epidemia } \\
\text { pela COVID-19. }\end{array}$ & $\begin{array}{l}\text { A telessaúde oferece capacidades para } \\
\text { triagem, cuidado e tratamento remotos, } \\
\text { auxilia o monitoramento, vigilância, } \\
\text { detecção e prevenção, e para a mitigação } \\
\text { dos impactos aos cuidados de saúde } \\
\text { indiretamente relacionados a COVID-19. }\end{array}$ \\
\hline A2 & $\begin{array}{l}\text { Produção de máscaras } \\
\text { cirúrgicas: estratégia no } \\
\text { combate à COVID-19 }\end{array}$ & Siman, et al. & $\begin{array}{l}\text { Relatar a experiência de } \\
\text { produção de máscaras } \\
\text { cirúrgicas por uma comissão de } \\
\text { produção de inovação } \\
\text { tecnológica. }\end{array}$ & $\begin{array}{l}\text { A produção chegou a } 1.300 \text { máscaras } \\
\text { cirúrgicas por dia. Todas passaram pelo } \\
\text { teste de qualidade em uma central de } \\
\text { distribuição antes de serem encaminhadas } \\
\text { aos serviços de saúde. }\end{array}$ \\
\hline A3 & $\begin{array}{l}\text { Tecnologias leves e } \\
\text { educação em saúde no } \\
\text { enfrentamento à } \\
\text { pandemia da COVID- } \\
19\end{array}$ & $\begin{array}{l}\text { Ceccon \& } \\
\text { Schneider. }\end{array}$ & $\begin{array}{l}\text { Discutir a importância das } \\
\text { tecnologias leves e da educação } \\
\text { em saúde no enfrentamento à } \\
\text { pandemia da COVID-19. }\end{array}$ & $\begin{array}{l}\text { É defendido que, em tempos de pandemia, é } \\
\text { fundamental a humanização do cuidado, o } \\
\text { diálogo e as relações de confiança entre } \\
\text { profissionais e usuários, que contribuem } \\
\text { para o enfrentamento à pandemia, } \\
\text { especialmente as tecnologias leves e a } \\
\text { educação em saúde. }\end{array}$ \\
\hline A4 & $\begin{array}{l}\text { Comparing South } \\
\text { Korea and Italy's } \\
\text { healthcare systems } \\
\text { and initiatives to } \\
\text { combat COVID-19 }\end{array}$ & $\begin{array}{c}\text { Palaniappan, et } \\
\text { al. }\end{array}$ & $\begin{array}{l}\text { Fazer uma comparação entre os } \\
\text { sistemas de saúde da Coreia do } \\
\text { Sul e da Itália e iniciativas } \\
\text { utilizadas para combater a } \\
\text { COVID-19. }\end{array}$ & $\begin{array}{l}\text { A Coreia do Sul utilizou a tecnologia e a } \\
\text { educação holística a seu favor, diminuindo } \\
\text { assim a transmissão da COVID-19. Já a } \\
\text { Itália é considerada o epicentro do surto na } \\
\text { Europa, devido as medidas de saúde } \\
\text { pública. }\end{array}$ \\
\hline A5 & $\begin{array}{l}\text { Applying the principles } \\
\text { of health technology } \\
\text { assessments to } \\
\text { intubation boxes for } \\
\text { patients with COVID-19 }\end{array}$ & Khemani, et al. & $\begin{array}{l}\text { Avaliar a segurança das caixas } \\
\text { de intubação usando os } \\
\text { componentes de um formulário } \\
\text { de Avaliação de Tecnologia em } \\
\text { Saúde. }\end{array}$ & $\begin{array}{l}\text { Na ausência de grandes ensaios clínicos ou } \\
\text { de um formulário de Avaliação de } \\
\text { Tecnologia em Saúde, propõe-se uma } \\
\text { abordagem baseada em evidências para } \\
\text { avaliar as caixas de intubação. }\end{array}$ \\
\hline A6 & $\begin{array}{l}\text { The need for fully bio- } \\
\text { based facemasks to } \\
\text { counter coronavirus } \\
\text { outbreaks: A } \\
\text { perspective }\end{array}$ & Das, et al. & $\begin{array}{l}\text { Apresentar a necessidade do } \\
\text { desenvolvimento de máscaras, } \\
\text { com matérias-primas e } \\
\text { processos alternativos para } \\
\text { conter surtos virais. }\end{array}$ & $\begin{array}{l}\text { O processo de desenvolvimento da máscara } \\
\text { promoverá o uso de recursos de base } \\
\text { biológica, reduzirá as emissões de gases de } \\
\text { efeito estufa e diminuirá a produção de } \\
\text { microplásticos que prejudicam o meio } \\
\text { ambiente. }\end{array}$ \\
\hline A7 & $\begin{array}{l}\text { Global Preparedness } \\
\text { Against COVID-19: We } \\
\text { Must Leverage the } \\
\text { Power of Digital Health }\end{array}$ & $\begin{array}{l}\text { Mahmood, et } \\
\text { al. }\end{array}$ & $\begin{array}{c}\text { Apresentar vários casos de uso } \\
\text { de aplicativos para controle de } \\
\text { infecção, diagnóstico e triagem } \\
\text { domiciliar, capacitação por } \\
\text { meio de informações, vigilância } \\
\text { de saúde pública e } \\
\text { epidemiologia. }\end{array}$ & $\begin{array}{l}\text { Foi identificado o acelerado uso e a adoção } \\
\text { de estratégias digitais, a fim de impactar } \\
\text { significativamente na COVID-19, bem } \\
\text { como em outras necessidades futuras de } \\
\text { cuidados de saúde. }\end{array}$ \\
\hline A8 & Uso de aplicaciones & Guisado- & Realizar uma revisão das & Das TICs observadas, são muitas as \\
\hline
\end{tabular}




\begin{tabular}{|c|c|c|c|c|}
\hline & $\begin{array}{c}\text { móviles y páginas web } \\
\text { para el diagnóstico de } \\
\text { la COVID-19 en } \\
\text { España }\end{array}$ & Clavero, et al. & $\begin{array}{l}\text { diferentes TICs, aplicativos e } \\
\text { páginas da web, visando a } \\
\text { gestão e / ou diagnóstico } \\
\text { presuntivo de COVID-19 na } \\
\text { Espanha. }\end{array}$ & $\begin{array}{c}\text { diferenças entre elas, tanto nas informações } \\
\text { recolhidas como nos recursos dedicados ao } \\
\text { cidadão. }\end{array}$ \\
\hline A9 & $\begin{array}{l}\text { Papel de la impresión } \\
3 \text { D para la protección } \\
\text { de los profesionales del } \\
\text { área quirúrgica y } \\
\text { críticos en la pandemia } \\
\text { de COVID-19 }\end{array}$ & Pedraja, et al. & $\begin{array}{l}\text { Descrever a experiência do } \\
\text { papel da impressão 3D em um } \\
\text { hospital terciário durante a } \\
\text { pandemia COVID-19. }\end{array}$ & $\begin{array}{c}\text { Foram produzidos os seguintes insumos } \\
\text { como dispositivos que auxiliam na proteção } \\
\text { dos fornecedores, produtos relacionados à } \\
\text { ventilação de pacientes infectados e swabs } \\
\text { orais e nasofaríngeos para identificação de } \\
\text { portadores de coronavírus com o objetivo } \\
\text { de traçar protocolos de ação em áreas } \\
\text { clínicas. }\end{array}$ \\
\hline A10 & $\begin{array}{l}\text { Estimando el número de } \\
\text { casos de COVID-19 } \\
\text { mediante una } \\
\text { herramienta web: } \\
\text { resultados de la } \\
\text { primera semana del } \\
\text { proyecto «Covid-19 } \\
\text { Trends» en Euskadi }\end{array}$ & Garitano, et al. & $\begin{array}{c}\text { Apresentar a análise da } \\
\text { ferramenta na comunidade } \\
\text { autônoma de Euskadi como um } \\
\text { estudo piloto durante a primeira } \\
\text { semana de aplicação do } \\
\text { questionário. }\end{array}$ & $\begin{array}{l}\text { Os casos estimados foram seis vezes mais } \\
\text { do que os RT-PCRs positivos para COVID- } \\
\text { 19. A incidência acumulada em } 14 \text { dias foi } \\
\text { de } 463,3 \text { por } 100.000 \text { habitantes, enquanto a } \\
\text { dos casos positivos por RT-PCR foi de } \\
139,6 \text { por } 100.000 \text { habitantes. }\end{array}$ \\
\hline A11 & $\begin{array}{c}\text { Explorando la } \\
\text { perspectiva de los } \\
\text { residentes sobre las } \\
\text { modalidades y } \\
\text { contenidos de } \\
\text { aprendizaje inteligente } \\
\text { para la educación } \\
\text { virtual de urología: } \\
\text { lección aprendida } \\
\text { durante la pandemia de } \\
\text { la COVID-19 }\end{array}$ & Campi, et al. & $\begin{array}{l}\text { Fornecer uma visão global da } \\
\text { opinião dos residentes de } \\
\text { urologia em todo o mundo } \\
\text { sobre essas tecnologias para } \\
\text { fins educacionais. }\end{array}$ & $\begin{array}{c}\text { Destes, } 78,4,78,2,56,9 \text { e } 51,9 \% \\
\text { consideraram os vídeos pré-gravados, } \\
\text { webinars interativos, podcasts e mídias } \\
\text { sociais, respectivamente, como } \\
\text { modalidades de aprendizagem inteligente } \\
\text { muito úteis. }\end{array}$ \\
\hline A12 & $\begin{array}{l}\text { La simulación como } \\
\text { herramienta para } \\
\text { facilitar la adaptación } \\
\text { de la organización } \\
\text { sanitaria a la pandemia } \\
\text { de COVID-19 }\end{array}$ & Maestre, et al. & $\begin{array}{l}\text { Descrever a experiência de um } \\
\text { centro de simulação para } \\
\text { contribuir como ferramenta } \\
\text { estratégica de apoio à mudança } \\
\text { dos serviços de saúde na } \\
\text { pandemia do COVID-19. }\end{array}$ & $\begin{array}{l}\text { São implementados três tipos de atividades } \\
\text { de treinamento de simulação: oficinas de } \\
\text { treinamento de formadores para proteção } \\
\text { contra transmissão por contato de queda, } \\
\text { simulações para proteção contra } \\
\text { transmissão de aerossol e um framework } \\
\text { para a análise de situações reais com } \\
\text { pacientes. }\end{array}$ \\
\hline A13 & $\begin{array}{l}\text { Covid-19 and digital } \\
\text { technology: mobile } \\
\text { applications available } \\
\text { for download in } \\
\text { smartphones }\end{array}$ & Neto, et al. & $\begin{array}{l}\text { Descrever aplicativos móveis } \\
\text { sobre COVID-19 disponíveis } \\
\text { para download em smartphones. }\end{array}$ & $\begin{array}{l}\text { Os aplicativos móveis sobre COVID-19 } \\
\text { encontravam-se disponíveis em países dos } \\
\text { cinco continentes, nos principais idiomas de } \\
\text { comunicação, de forma gratuita, entretanto, } \\
\text { com escassa acessibilidade para pessoas } \\
\text { com deficiência. }\end{array}$ \\
\hline
\end{tabular}

Fonte: Autores da pesquisa (2020).

Os 13 estudos foram divididos em 4 categorias com base no título e no assunto abordado (Tabela 2), para que pudessem ser melhor explorados. 
Tabela 2. Categorização dos artigos.

\begin{tabular}{|l|l|}
\hline $\begin{array}{l}\text { Fabricação e avaliação de insumos por meio de } \\
\text { tecnologias }\end{array}$ & A1, A6, A13 \\
\hline $\begin{array}{l}\text { Utilização de aplicativos e a telessaúde em meio a } \\
\text { pandemia }\end{array}$ & A2, A7, A8, A10 \\
\hline $\begin{array}{l}\text { Utilização de tecnologias como forma de } \\
\text { educação em saúde }\end{array}$ & $\mathrm{A} 3, \mathrm{~A} 4, \mathrm{~A} 12$ \\
\hline Impressão 3D, tubos e testes sorológicos & $\mathrm{A} 5, \mathrm{~A} 9, \mathrm{~A} 11$ \\
\hline
\end{tabular}

Fonte: Autores (2020).

Os estudos trouxeram abordagens amplas e diferentes nas pesquisas sobre tecnologias e COVID-19, desde os títulos até a abordagem metodológica. No que se diz respeito a esse aspecto, o processo de metodologia dos estudos apresentou-se em grande parte em pesquisas pilotos (4), relato de experiências (3), estudos quantitativos (2), e 1 de cada dos seguintes: transversal, documental, comparativo, descritivo exploratório e ensaio reflexivo. Ressalta-se que alguns estudos trouxeram mais de um tipo de abordagem.

Em relação ao local de publicação e pesquisas dos estudos, 5 destes são do Brasil, 4 de forma Global, 3 da Espanha e da Coreia do Sul. Importante ressaltar a influência da COVID-19 em todo o mundo, fazendo com que diversos países investissem e pesquisassem o tema.

Com a carência de tecnologias farmacológicas ou imunobiológicas (ex.: soros e vacinas) com eficácia comprovada contra o Sars-CoV-2, outras tecnologias de controle e propagação da epidemia tornaram-se fundamentais. Nesta perspectiva, a criação e adaptação de aplicativos móveis tornaram-se tecnologias digitais inovadoras no combate à epidemia da COVID-19 no mundo (Casas, et al., 2020).

Diversas são as tecnologias criadas seja para diagnóstico ou para qualificação profissional. A impressão 3D foi utilizada por diversos pesquisadores e produtores, bem como o da indústria em saúde. Foram produzidos insumos como dispositivos que auxiliam na proteção dos fornecedores, produtos relacionados à ventilação de pacientes infectados e swabs orais e nasofaríngeos para identificação de portadores de coronavírus com o objetivo de traçar protocolos de ação em áreas clínicas, quando isso não se fez sozinho cabível, entraram as demais tecnologias (Pedraja, et al., 2020).

Neto et al. (2020), apresenta, em seu estudo transversal, 52 aplicativos móveis sobre COVID-19, obtidos nas lojas virtuais para smartphones com sistema operacional Android e iOS. Destes, 41 (78,9\%) eram destinados ao público em geral, seis $(11,5 \%)$ para profissionais de saúde e cinco (9,6\%) para pacientes com diagnóstico de COVID-19. Os principais temas abordados tratavam sobre sintomas da doença, prevenção e cuidados contra a contaminação, além de disponibilizarem monitoramento de casos suspeitos e confirmados. No que diz respeito ao país, o Brasil desenvolveu 15 (28,9\%) aplicativos, sendo a nação com maior número de aplicativos sobre COVID-19.

Corroborando com o estudo, Guisado-Clavero et al. (2020), analisou seis aplicativos móveis correspondentes a cinco comunidades autônomas e uma do Ministério da Saúde da Espanha, além de quatro páginas da web das Secretarias de Saúde das comunidades autônomas correspondentes. Nesta pesquisa foi possível identificar o potencial dessas ferramentas para detecção de possíveis casos, acompanhamento, estudo de contatos com o caso suspeito e, sobretudo, prevenção das complicações através do diagnóstico precoce. Todavia, destaca-se também a importância de validação para generalizar seu uso por profissionais da saúde no acompanhamento dos seus pacientes.

No que diz respeito à vigilância epidemiológica, muitos aplicativos e sites foram criados e desenvolvidos voltados para o combate da pandemia. Estes se utilizam de tecnologias como geolocalização, registro de contatos via Bluetooth, big data 
e inteligência artificial para produzir informações que contribuam no enfrentamento da crise de saúde de forma mais eficiente (Cascón-Katchadourian, 2020).

Nesta perspectiva, a Comunidade Autónoma do País Basco, Espanha, com o objetivo de estimar o número de casos sintomáticos da doença e gerar informações úteis para a tomada de decisão no contexto da pandemia, criaram um questionário anônimo da web, disponibilizado através das redes sociais, com informações epidemiológicas relacionadas à situação de saúde. Esses dados coletados foram comparados com os casos confirmados através do exame de diagnóstico da COVID-19 (RTPCR). Em uma semana, o questionário foi respondido por 128.182 pessoas (5,5\% da população basca), destes, 27.599 atenderam à definição de caso. Os casos estimados foram seis vezes mais do que os RT-PCRs positivos para COVID-19. A partir da pesquisa em questão foi possível identificar que a criação e implementação de um sistema de vigilância sindrômica é possível, barato, rápido e aplicável em qualquer lugar usando internet e redes sociais, principalmente em países de renda média e baixa, onde a disponibilidade de testes diagnósticos é escassa (Garitano, et al., 2020).

Em outra vertente está a telessaúde que incorpora o uso de tecnologias de informação e telecomunicação objetivando a promoção de cuidados em saúde à distância, difusão de informações, bem como oferta de serviços e atividades de treinamento para os profissionais da assistência. Durante os surtos anteriores, como COV da síndrome respiratória do Oriente Médio (Middle East Respiratory Syndrome Coronavirus, MERS-CoV), Ebola e Zika, a telessaúde teve sua parcela de contribuição na redução da transmissão viral por meio da implantação de plano de ação composto por monitoração remota de pacientes, teleconsultoria, além de teleconsultas, as quais possibilitaram a triagem dos casos e o atendimento de indivíduos isolados (Carvalho \& Teixeira, 2020).

Caetano et al. (2020), discute em seu estudo a contribuição da telessaúde para enfrentamento da COVID-19, bem como as iniciativas recentes do Brasil em consolidar a telemedicina com vista a aperfeiçoar o Sistema Único de Saúde (SUS). Dentre o escopo de atividades estão a teleconsultoria, telediagnóstico, telemonitoramento, telerregulação, teleeducação, segunda opinião formativa e teleconsulta. Assim, a telessaúde se mostra como um recurso importante, visto a sua capacidade de reduzir a circulação de indivíduos nos serviços de saúde, diminuir o risco de contaminação e propagação da doença, bem como chegar a lugares de difícil acesso ou com estrutura deficitária.

Além disso, a Organização Mundial da Saúde (OMS) recomendou que casos suspeitos de COVID-19 com sintomas leves e sem comorbidades associadas poderiam ser tratados em casa com monitoramento clínico cuidadoso. Nessa perspectiva, a teleconsulta tem ampla recomendação, pois previne sobrecarga dos serviços de saúde, bem como minimiza os riscos de adoecimentos dos profissionais de saúde, duas situações que podem colocar em risco os sistemas de saúde, principalmente de países de média e baixa renda. Destaca-se também o custo operacional para funcionamento de centros de telemedicina, visto que estes podem ser menores em relação ao funcionamento de unidades hospitalares, por exemplo (Mahmood et al., 2020).

Sabendo que a transmissão de COVID-19 pode ocorrer através da tosse ou gotículas respiratórias, foi necessário adotar a utilização de equipamentos de proteção individual (EPI’s), como as máscaras faciais, para conter a dispersão do vírus. Entretanto, houve uma escassez de EPI's disponíveis para profissionais da área da saúde, com isso montou-se uma estratégia de produção de máscaras cirúrgicas para suprir essa demanda. No total, foram produzidas 63 mil máscaras cirúrgicas, com custo-benefício satisfatório. Todas passaram pelo teste de qualidade em uma central de distribuição antes de serem doadas para hospitais e outros serviços de saúde (Siman, et al., 2020).

Seguindo por um caminho sustentável, Das et al. (2020), realizaram pesquisas para desenvolver máscaras de proteção através de biopolímero de glúten de trigo, que é um subproduto ou co-produto das indústrias de cereais. Em seus estudos foi possível verificar que o plástico formado a partir do glúten pode ser resistente a micróbios. A elaboração dessa máscara pode simultaneamente ser eficaz na redução da transmissão de doenças infecciosas ao mesmo tempo em que vai promover o uso de 
recursos de base biológica, reduzirá as emissões de gases de efeito estufa e diminuirá a produção de microplásticos que prejudicam o meio ambiente.

Além da utilização e fabricação de mascaras como dispositivos de proteção individual, houveram ainda a criação de outras formas de EPI's, visto a necessidade de uma proteção larga e que protegesse amplamente do vírus. Uma delas foram as caixas de intubação que são um novo dispositivo médico que podem prevenir potencialmente a transmissão de COVID-19 para profissionais de saúde (Khemani, et al., 2020)

Além de disponibilizar os EPI's, foi indispensável adotar novas medidas de treinamento seguro e eficaz, para os profissionais da área da saúde. Como ferramenta, foi utilizado um centro de simulações, onde foi possível realizar três tipos de atividades: Oficinas de capacitação na colocação e retiradas de EPI's, para evitar a contaminação por contato direto e por aerossóis, além de promover estrutura para apoio aos profissionais durante o dia, para receber a equipe com empatia e motivála. O que facilitou a adaptação por parte desses profissionais no combate a COVID-19 (Maestre, et al., 2020).

A educação profissional também se torna importante nesse momento, visto que atualizações são constantes. Uma pesquisa realizada com profissionais residentes da saúde em todo o mundo mostrou que destes 78,4\% consideraram vídeos prégravados, webinars interativos, podcasts e mídias sociais, respectivamente, como modalidades de aprendizagem inteligente muito úteis para repasse de capacitações sobre a COVID-19 (Campi, et al., 2020).

Nenhuma tecnologia pode ser essencial sem a utilização correta das mesmas tanto por parte da população como dos profissionais da saúde. Além disso ressalta-se a importância da criação de vínculo entre essas partes, pois quando há geração de confiança a possibilidade de seguir as indicações profissionais são maiores. Em tempos de isolamento, esse contato tornouse mais difícil, tendo a tecnologia papel de importância na aproximação da saúde e população (Ceccon \& Schneider, 2020).

Um estudo realizado na Coreia do Sul e na Itália de forma comparativa mostrou que a Coreia do Sul utilizou a tecnologia e a educação holística de sua comunidade médica, implementando centros de rastreio para pessoas com suspeita de infecção sem sair do carro, sabendo-se que nesse momento o contato próximo era o menos indicado. Dessa forma entende-se a importância da educação da sociedade. Por outro lado, a Itália, que foi um dos países que mais sofreu com a pandemia, deveuse em parte à natureza reativa das medidas de saúde pública no país em comparação com a resposta pró-ativa da Coreia do Sul (Palaniappan, et al., 2020).

\section{Considerações Finais}

A pandemia e a crise no setor saúde evidenciada com o COVID-19 serviram de campo fértil para a criação e adaptação de tecnologias para auxiliar no enfrentamento dos casos, tornando-se fundamentais para a prática assistencial. Nesta perspectiva, surgiram ferramentas inovadoras para o combate à epidemia da COVID-19 em todo o mundo.

Assim, torna-se possível identificar e associar o potencial de aplicação que a tecnologia apresenta, seja na detecção dos casos, proteção, atendimento e, sobretudo, diagnóstico precoce, gerando informações úteis para a tomada de decisão de forma mais eficiente no contexto da pandemia.

Sugere-se a partir dos dados aqui expostos, que mais estudos na temática sejam realizados, tendo em vista a escassez de estudos e o período de transição vivido atualmente, onde as tecnologias desenvolvidas, ainda podem se ampliar ou se atenuar, dependendo das demandas da população.

\section{Referências}

Almeida, B. A., Doneda, D., Ichihara, M. Y., Barral-Netto, M., Matta, G. C., Rabello, E. T., Gouveia, F. C. \& Barreto, M. (2020). Preservação da privacidade no enfrentamento da COVID-19: dados pessoais e a pandemia global. Ciênc. saúde coletiva, 25(supl. 1), $2487-2492$.

Beaunoyer, E., Dupéré, S., \& Guitton, M. J. (2020). COVID-19 and digital inequalities: Reciprocal impacts and mitigation strategies. Elsevier, 111, e106424. 
Caetano, R., Silva, A. B., Guedes, A. C. C. M., Paiva, C. C. N., Ribeiro, G. R., Santos, D. L. \& Silva, R. M. (2020). Desafios e oportunidades para telessaúde em tempos da pandemia pela COVID-19: uma reflexão sobre os espaços e iniciativas no contexto brasileiro. Cadernos de Saúde Pública, 36(5), e00088920.

Campi, R., Amparore, D., Checcucci, E., Claps, F., Teoh, J. Y. C., Serni, S., Scarpa, R. M., Porpiglia, F., Carrion, D. M., Rivas, J. G., Loeb, S., Cacciamani, G. E. \& Esperto, F. (2021). Explorando la perspectiva de los residentes sobre las modalidades y contenidos de aprendizaje inteligente para la educación virtual de urología: lección aprendida durante la pandemia de la COVID-19. Actas Urológicas Españolas, 45(1), 39-48.

Carvalho, W., \& Teixeira, L. A. (2020). Telessaúde e COVID-19: estratégia de combate à pandemia e um novo caminho para o cuidado em saúde. InterAmerican Journal of Medicine and Health, 3.

Casas, C. P. R., Silva, J., Castro, R., Ribeiro-Alves, M., \& Franco, C. M. (2020). Avaliação de tecnologias em saúde: tensões metodológicas durante a pandemia de COVID-19. Estudos Avançados, 34(99), 77-96.

Cascón-Katchadourian, Jesús-Daniel. (2020). Tecnologías para luchar contra la pandemia Covid-19: geolocalización, rastreo, big data, SIG, inteligencia artificial y privacidad. Profesional de la información, 29(4).

Ceccon, R. F., \& Schneider, I. J. C. (2020). Tecnologias leves e educação em saúde no enfrentamento à pandemia da COVID-19. Scientific Electronic Library Online Prepints, 1.

Coelho, A. L., Morais, I. A., \& Rosa, W. V. S. (2020). A utilização de tecnologias da informação em saúde para o enfrentamento da pandemia do COVID-19 no Brasil. Cadernos Ibero-Americanos de Direito Sanitário, 9(3), 183-199.

Das, O., Neisiany, R. E., Capezza, A. J., Hedenqvist, M. S., Foorsht, M., Xu, Q., Jiang, L., Ji, D., Ramakrishna, S. (2020). The need for fully bio-based facemasks to counter coronavirus outbreaks: A perspective. Rev. Science of The Total Environment, 1(736), e139611.

Garitano, I., Linares, M., Santos, L., Santamaría, V., Galicia, F., \& Ramos, J. M. (2020). Estimando el número de casos de COVID-19 mediante una herramienta web: resultados de la primera semana del proyecto «Covid-19 Trends» en Euskadi. Medicina de Família - Semergen, $46(1), 111-117$.

Guisado-Clavero, M., Ares-Blanco, S., \& Abdellah, L. D. B. (2020). Uso de aplicaciones móviles y páginas web para el diagnóstico de la COVID-19 en España. Enfermedades Infecciosas y Microbiologia Clınica.

Júnior, J. H. S., Raasch, M., \& Soares, J. C. (2020). Da Desinformação ao Caos: uma análise das Fake News frente à pandemia do Coronavírus (COVID-19) no Brasil. Cadernos de Prospecção - Salvador, 13 (2), 331-346.

Khemani, E., Lampitoc, M., \& Duvall, D. (2020). Aplicação dos princípios de avaliações de tecnologia de saúde a caixas de intubação para pacientes com COVID-19. BMJ Open Quality, 9, e001010.

Maestre, J. M., Rábago, J. L., Cimadevilla, B., Pedraja, J., Moral, I., \& Manuel-Palazuelos, J. C. (2020). La simulación como herramienta para facilitar la adaptación de la organización sanitaria a la pandemia de COVID-19. Rev. Educación médic, 1(36), 1-6.

Mahmood, S., Hasan, K., Carras, M. C., \& Labrique, A. (2020). Global Preparedness Against COVID-19: We Must Leverage the Power of Digital Health. JMIR Public Health Surveill, 6 (2), e18980.

Mendes, K. D. S., Silveira, R. C. C. P., \& Galvão, C. M. (2008). Revisão integrativa: método de pesquisa para a incorporação de evidências na saúde e na enfermagem. Texto \& Contexto - Enfermagem, 17(4), 758-764.

Moher, D., Liberati, A., Tetzlaff, J., Altman, D. G. (2009). Preferred Reporting Items for Systematic Reviews and Meta Analyses: The PRISMA Statement. PLoS Medicine, 6(7), e1000097.

Neto, N. M. G., Sá, G. G. M., Barbosa, L. U., Pereira, J. C. N., Henriques, A. H. B., \& Barros, L. M. (2020). COVID-19 and digital technology: mobile applications available for download in smartphones. Texto \& Contexto - Enfermagem, 29, e20200150.

Palaniappan, A., Dave, U., \& Gosine, B. (2020). Comparing South Korea and Italy's healthcare systems and initiatives to combat COVID-19. Rev. Panam. Salud Publica, 44, e53.

Pedraja, J., Maestre, J. M., Rabanal, J. M., Morales, C., Aparicio, J., \& Del Moral, I. (2020). O papel da impressão 3D para a proteção de profissionais cirúrgicos e de cuidados intensivos na pandemia de COVID-19. Rev Esp Anestesiol Reanim, 67(8), 417-424.

Pereira, A. S., Shitsuka, D. M., Parreira, F. J., \& Shitsuka, R. (2018). Metodologia da pesquisa científica. Santa Maria, RS: Núcleo de Tecnologia Educacional da Universidade Federal de Santa Maria.

Pereira, M. D., Oliveira, L. C., Costa, C. F. T., Bezerra, C. M. O., Pereira, M. D., Santos, C. K. A., \& Dantas, E. H. M. (2020). The COVID-19 pandemic, social isolation, consequences on mental health and coping strategies: an integrative review. Research, Society and Development, 9 (7), e652974548.

Siman, A.G., Diaz, F. B. B. S., Braga, L. M., Correia, M. D. L., Ayres, L. F. A., \& Cunha, S. G. S. (2020). Produção de máscaras cirúrgicas: estratégia no combate à covid-19. Rev. Baiana enferm., 34, e37234.

Souza, M. T., Silva, M. D., \& Carvalho, R. (2010). Revisão integrativa: o que é e como fazer? Rev. Einstein, 8(1), $102-106$. 